PROCEEDINGS OF THE

AMERICAN MATHEMATICAL SOCIETY

Volume 135, Number 6, June 2007, Pages 1915-1922

S 0002-9939(07)08757-6

Article electronically published on February 6, 2007

\title{
DYNAMIC APPROACH TO A STOCHASTIC DOMINATION: THE FKG AND BRASCAMP-LIEB INEQUALITIES
}

\author{
TADAHISA FUNAKI AND KOU TOUKAIRIN
}

(Communicated by Edward C. Waymire)

\begin{abstract}
A coupling based on a pair of stochastic differential equations is introduced to show a stochastic domination for a system with continuous spins, from which the FKG and Brascamp-Lieb like inequalities follow.
\end{abstract}

\section{INTRODUCTION}

The FKG inequality and Brascamp-Lieb momentum inequality have their origins in statistical mechanics or quantum field theory, and play an important role as basic tools. The former implies positive correlations under the Gibbs measures with attractive interaction potentials; see [2], 4], 6], 9], 12, [13. The latter shows that the centered moments of a distribution having a log-concave density with respect to a Gaussian measure $\mu$ are not larger than those of $\mu$; see [3], 4, 7. The Gaussian measure represents a free field or a harmonic crystal in physics.

The purpose of this paper is to give a new proof for these inequalities or their variants, especially in the case of continuous spins, based on a stochastic domination which is shown via a coupling of stochastic differential equations with the help of an ergodic theorem. A similar idea was employed by Holley [9] to prove the FKG inequality in the case of discrete spins due to a coupling of Markov chains. Bakry and Michel [2] gave another dynamic proof of the FKG inequality for a system with continuous spins. On the other hand, Brascamp-Lieb inequality was recently extended by Caffarelli [4] with a deep insight in optimal mass transportation and using the Monge-Ampére equation (cf. [1). Our approach is different, much simpler (at least for probabilists) and applicable to the derivation of certain related inequalities.

We shall work on the $d$-dimensional Euclidean space $\mathbb{R}^{d}$, which represents the configuration space of continuous spins on $d$ sites. The class of all Borel probability measures on $\mathbb{R}^{d}$ is denoted by $\mathcal{P}\left(\mathbb{R}^{d}\right)$. The space $\mathbb{R}^{d}$ is equipped with a natural partial order $x \leq y$ for $x=\left(x_{i}\right)_{i=1}^{d}, y=\left(y_{i}\right)_{i=1}^{d} \in \mathbb{R}^{d}$ defined by $x_{i} \leq y_{i}$ for every $1 \leq i \leq d$. For $\mu, \nu \in \mathcal{P}\left(\mathbb{R}^{d}\right)$, we say $\nu$ stochastically dominates $\mu$ and write $\mu \leq \nu$ if $E^{\mu}[F] \leq E^{\nu}[F]$ holds for all bounded non-decreasing (in the above partial order) functions $F$ on $\mathbb{R}^{d}$. Note that the stochastic domination $\mu \leq \nu$ is equivalent to

Received by the editors April 10, 2006.

2000 Mathematics Subject Classification. Primary 82B31; Secondary 82B20, 60K35.

Key words and phrases. Stochastic domination, FKG inequality, Brascamp-Lieb inequality, Coupling.

The first author was supported in part by JSPS Grants (B)14340029 and 17654020 .

(c) 2007 American Mathematical Society Reverts to public domain 28 years from publication 
the existence of two $\mathbb{R}^{d}$-valued random variables $X$ and $Y$, which are realized on a common probability space, being distributed under $\mu$ and $\nu$ (we denote $X \sim \mu$ and $Y \sim \nu$ ), respectively, and satisfying $X \leq Y$ a.s.; see Theorem 2.4 on p. 72 of [12] (at least for compact state spaces; an extension to $\mathbb{R}^{d}$ is easy).

Section 2 shows Holley's stochastic domination and the FKG inequality as its corollary under a continuous setting. Section 3 discusses the domination to the origin and Brascamp-Lieb type inequalities. The Gaussian property of $\mu$ is not assumed in general. The convexity of potentials is also relaxed. Section 4 proposes an extension of the Brascamp-Lieb inequality for a genuinely non-convex potential obtained as a small perturbation of a convex one, which includes a potential of double-well type when $d=1$.

\section{Holley's Stochastic domination and FKG INEQUALITy}

Holley [9] gave a sufficient condition for the stochastic domination and applied it to show the FKG inequality under a discrete setting; see also Chapter II-2 of [12. It was then generalized by Preston 13 including the case of continuous spins. We use a dynamic approach based on stochastic differential equations to establish Holley's stochastic domination under a continuous setting.

Let $\mu$ and $\nu \in \mathcal{P}\left(\mathbb{R}^{d}\right)$ be probability measures of the following forms:

$$
\begin{aligned}
& \mu(d x)=\frac{1}{Z_{\mu}} e^{-V(x)} d x, \\
& \nu(d x)=\frac{1}{Z_{\nu}} e^{-V(x)-U(x)} d x,
\end{aligned}
$$

where $Z_{\mu}$ and $Z_{\nu}$ are finite normalizing constants, and the measurable functions $V$ and $U$ on $\mathbb{R}^{d}$ satisfy the following condition:

$$
V(x)+V(y)-V(x \vee y)-V(x \wedge y) \geq U(x \vee y)-U(x),
$$

for all $x, y \in \mathbb{R}^{d}$, where $x \vee y=\left(x_{i} \vee y_{i}\right)_{i=1}^{d}$ and $x \wedge y=\left(x_{i} \wedge y_{i}\right)_{i=1}^{d}$. In general, for $\mu$ and $\nu \in \mathcal{P}\left(\mathbb{R}^{d}\right)$ being absolutely continuous with respect to $d x$, the condition (2.2) is equivalent to Holley's condition (cf. [13]):

$$
g(x \vee y) f(x \wedge y) \geq g(x) f(y),
$$

where $f$ and $g$ are the probability density functions of $\mu$ and $\nu$, respectively.

Proposition 2.1. Assume that $V, U \in C^{1}\left(\mathbb{R}^{d}\right)$ and $\nabla V, \nabla U$ are both Lipschitz continuous. Consider the pair of stochastic differential equations on $\mathbb{R}^{d}$ :

$$
\begin{aligned}
d X_{t} & =-\frac{1}{2} \nabla V\left(X_{t}\right) d t+d w_{t}, \\
d Y_{t} & =-\frac{1}{2}\left\{\nabla V\left(Y_{t}\right)+\nabla U\left(Y_{t}\right)\right\} d t+d w_{t},
\end{aligned}
$$

driven by a common d-dimensional Brownian motion $w=\left\{w_{t} ; 0 \leq t<\infty\right\}$. Then, $X_{0} \leq Y_{0}$ implies $X_{t} \leq Y_{t}$ for all $t \geq 0$, a.s. 
Proof. Since $X_{0} \leq Y_{0}$, we have by Itô's formula

$$
\begin{aligned}
\phi_{t} & \equiv \sum_{i=1}^{d}\left(X_{t}^{i}-Y_{t}^{i}\right)^{2} 1_{\left\{X_{t}^{i}>Y_{t}^{i}\right\}} \\
& =\sum_{i=1}^{d} \int_{0}^{t}\left(X_{s}^{i}-Y_{s}^{i}\right) 1_{\left\{X_{s}^{i}>Y_{s}^{i}\right\}}\left(\nabla_{i} V\left(Y_{s}\right)+\nabla_{i} U\left(Y_{s}\right)-\nabla_{i} V\left(X_{s}\right)\right) d s,
\end{aligned}
$$

where $X_{t}=\left(X_{t}^{i}\right)_{i=1}^{d}$ and $\nabla_{i} V=\partial V / \partial x_{i}$, etc. However, on $\left\{x_{i}>y_{i}\right\}$, we have

$$
\begin{aligned}
& \nabla_{i} V(y)+\nabla_{i} U(y)-\nabla_{i} V(x) \\
& \leq \nabla_{i} V(y)+\nabla_{i} U(y)-\nabla_{i} V(x \vee y)-\nabla_{i} U(x \vee y) \\
& =\sum_{k=1}^{d}\left\{\nabla_{i} V\left((x \vee y)^{(k+1)}\right)-\nabla_{i} V\left((x \vee y)^{(k)}\right)\right. \\
& \left.\quad+\nabla_{i} U\left((x \vee y)^{(k+1)}\right)-\nabla_{i} U\left((x \vee y)^{(k)}\right)\right\} 1_{\left\{x_{k}>y_{k}\right\}} \\
& \leq C \sum_{k=1}^{d}\left(x_{k}-y_{k}\right) 1_{\left\{x_{k}>y_{k}\right\}},
\end{aligned}
$$

where $(x \vee y)^{(k)}, 1 \leq k \leq d+1$, are defined by

$$
\left((x \vee y)^{(k)}\right)_{j}=x_{j} \vee y_{j}(\text { for } j \geq k), y_{j}(\text { for } j \leq k-1) .
$$

Indeed, for the second line, we have used the condition (2.2) which implies

$U\left(\left(x+\epsilon e^{i}\right) \vee y\right)-U(x \vee y) \leq\left(V\left(x+\epsilon e^{i}\right)-V(x)\right)-\left(V\left(\left(x+\epsilon e^{i}\right) \vee y\right)-V(x \vee y)\right)$

for $\epsilon>0$ on $\left\{x_{i}>y_{i}\right\}$; take $x+\epsilon e^{i}$ and $x \vee y$ for $x$ and $y$, respectively, in (2.2), where $e^{i} \in \mathbb{R}^{d}$ stands for the $i$ th unit vector. For the third line, note that $(x \vee y)^{(1)}=x \vee y$, $(x \vee y)^{(d+1)}=y$ and $x_{k} \leq y_{k}$ implies $(x \vee y)^{(k+1)}=(x \vee y)^{(k)}$. The last line follows from the Lipschitz continuity of $\nabla V$ and $\nabla U$. Substituting (2.5) for (2.4), we have

$$
\begin{aligned}
\phi_{t} & \leq C \sum_{i=1}^{d} \int_{0}^{t}\left(X_{s}^{i}-Y_{s}^{i}\right) 1_{\left\{X_{s}^{i}>Y_{s}^{i}\right\}} \sum_{k=1}^{d}\left(X_{s}^{k}-Y_{s}^{k}\right) 1_{\left\{X_{s}^{k}>Y_{s}^{k}\right\}} d s \\
& \leq C d \int_{0}^{t} \sum_{i=1}^{d}\left(X_{s}^{i}-Y_{s}^{i}\right)^{2} 1_{\left\{X_{s}^{i}>Y_{s}^{i}\right\}} d s \\
& =C d \int_{0}^{t} \phi_{s} d s .
\end{aligned}
$$

This implies $\phi_{t}=0$ with the help of Gronwall's lemma, and the proof is complete.

The coupling introduced in Proposition 2.1 deduces Holley's stochastic domination for continuous spins:

Theorem 2.2. Let $\mu, \nu \in \mathcal{P}\left(\mathbb{R}^{d}\right)$ be the probability measures satisfying the condition (2.2) or equivalently (2.3). Then $\nu$ stochastically dominates $\mu$. 
Proof. The probability measures $\mu$ and $\nu$ can be approximated weakly by sequences $\left\{\mu_{n} \in \mathcal{P}\left(\mathbb{R}^{d}\right)\right\}_{n=1,2, \ldots}$ and $\left\{\nu_{n} \in \mathcal{P}\left(\mathbb{R}^{d}\right)\right\}_{n=1,2, \ldots}$ having the forms:

$$
\begin{aligned}
& \mu_{n}(d x)=\frac{1}{Z_{\mu_{n}}} e^{-V_{n}(x)} d x, \\
& \nu_{n}(d x)=\frac{1}{Z_{\nu_{n}}} e^{-V_{n}(x)-U_{n}(x)} d x,
\end{aligned}
$$

respectively, where $V_{n}$ and $U_{n}$ satisfy (2.2) together with the conditions for $V$ and $U$ stated in Proposition 2.1. We can find such $V_{n}$ and $U_{n}$ by smearing $V$ and $U$, respectively; note that condition (2.2) is not harmed under the convolutions. Once Theorem 2.2 is shown for $\mu_{n}$ and $\nu_{n}$ instead of $\mu$ and $\nu$, one can prove it for general $\mu$ and $\nu$ by taking the limit.

Let $X_{t}$ and $Y_{t}$ be as in Proposition 2.1 satisfying $X_{0}=Y_{0}$. Then $X_{t} \leq Y_{t}$ holds for all $t \geq 0$, a.s. But $\mu$ and $\nu$ are invariant (in fact, reversible) measures for $X_{t}$ and $Y_{t}$, respectively (see [5], 10, [14] for example), and the ergodicity shows that the distributions on $\mathbb{R}^{d}$ of $X_{t}$ and $Y_{t}$ weakly converge to $\mu$ and $\nu$, respectively, as $t \rightarrow \infty$. The proof is concluded by letting $t \rightarrow \infty$ in the inequalities $E\left[F\left(X_{t}\right)\right] \leq E\left[F\left(Y_{t}\right)\right]$, which hold for all bounded continuous non-decreasing functions $F$ on $\mathbb{R}^{d}$.

The FKG inequality follows immediately from Theorem 2.2 .

Corollary 2.3. Let $f$ be a probability density function on $\mathbb{R}^{d}$ satisfying

$$
f(x \vee y) f(x \wedge y) \geq f(x) f(y),
$$

for all $x, y \in \mathbb{R}^{d}$, and let $\mu(d x)=f(x) d x \in \mathcal{P}\left(\mathbb{R}^{d}\right)$. Then, for all non-decreasing functions $F$ and $G$ on $\mathbb{R}^{d}$, we have

$$
E^{\mu}[F ; G]=E^{\mu}[F G]-E^{\mu}[F] E^{\mu}[G] \geq 0 .
$$

Proof. The proof is standard. Indeed, without loss of generality, we may assume $G>0$ (by approximating it by functions bounded below and adding a constant) and apply Theorem 2.2 with the choice of $f(x)=e^{-V(x)}$ and $G(x)=e^{-U(x)}$. Note that (2.6) implies $V(x)+V(y)-V(x \vee y)-V(x \wedge y) \geq 0$ and therefore the condition (2.2).

Remark 2.4. Corollary 2.3 can be directly shown via the coupling introduced in the proof of Proposition 2.1. In fact, noting that $\nabla_{i} U \leq 0$, the estimate (2.5) on $\left\{x_{i}>y_{i}\right\}$ may be replaced by a simpler one:

$$
\begin{aligned}
& \nabla_{i} V(y)+\nabla_{i} U(y)-\nabla_{i} V(x) \\
& \quad \leq \sum_{k=1}^{d}\left\{\nabla_{i} V\left((x \vee y)^{(k+1)}\right)-\nabla_{i} V\left((x \vee y)^{(k)}\right)\right\} 1_{\left\{x_{k}>y_{k}\right\}} \\
& \quad \leq C \sum_{k=1}^{d}\left(x_{k}-y_{k}\right) 1_{\left\{x_{k}>y_{k}\right\}} .
\end{aligned}
$$

\section{Stochastic domination and Brascamp-Lieb type inequalities}

Brascamp and Lieb 3 ] proved an inequality for a concentration of random variables around their means. The following theorem is in the form extended by Caffarelli [4]: 
Theorem 3.1. Let $\mu \in \mathcal{P}\left(\mathbb{R}^{d}\right)$ be a centered Gaussian measure and let

$$
\nu(d x)=\frac{1}{Z_{\nu}} e^{-U(x)} \mu(d x) \in \mathcal{P}\left(\mathbb{R}^{d}\right),
$$

be normalizable, i.e., $Z_{\nu}=\int_{\mathbb{R}^{d}} e^{-U(x)} \mu(d x)<\infty$. Then, if $U: \mathbb{R}^{d} \rightarrow \mathbb{R}$ is convex, for every $v \in \mathbb{R}^{d}$ and $\psi: \mathbb{R} \rightarrow \mathbb{R}$, convex and bounded below, we have

$$
E^{\nu}\left[\psi\left((v, Y)-E^{\nu}[(v, Y)]\right)\right] \leq E^{\mu}[\psi((v, X))],
$$

where $X \sim \mu, Y \sim \nu$ and $(v, X)$ denotes the inner product of $v$ and $X$ in $\mathbb{R}^{d}$.

This theorem was first proved by Brascamp and Lieb 3] for $\psi(a)=|a|^{p}, p \geq 1$. Giacomin [7] showed a related stochastic domination:

Theorem 3.2. Let $\mu$ and $\nu \in \mathcal{P}\left(\mathbb{R}^{d}\right)$ be as in Theorem 3.1. Then one can construct two $\mathbb{R}^{d}$-valued random variables $X$ and $Y$ such that $X \sim \mu, Y \sim \nu$ and a stochastic domination

$$
\left[(v, Y)-M_{v}\right]_{+} \leq[(v, X)]_{+}, \quad \text { a.s. }
$$

holds for every $v \in \mathbb{R}^{d}$, where $[a]_{+}=\max \{a, 0\}$ and $M_{v}$ denotes the median of $(v, Y)$.

Our goal is to extend the Brascamp-Lieb inequality in a spirit of stochastic domination. Our result does not completely cover Theorem 3.1, but, in a sense, it is more general. For instance, we only require the radial symmetry of $\mu$, and its Gaussian property is unnecessary in Theorem 3.3. We also notice that conditions (3.2) or (3.4) required below for the potential $U$ is slightly different from conventional one; in our setting, it need not be convex. We can apply the following theorems, e.g., for a square-well potential, which is non-convex.

In the case that $\mu$ is Gaussian, the argument by Prékopa and Leindler (for marginal distributions) actually reduces the proof of Theorem 3.1 to the onedimensional case; see [3], 4, 7]. From this viewpoint, the following theorem, stated generally on $d$ dimensional spaces, might have its own interest already when $d=1$.

Theorem 3.3. Let $\mu \in \mathcal{P}\left(\mathbb{R}^{d}\right)$ be radially symmetric and let $\nu \in \mathcal{P}\left(\mathbb{R}^{d}\right)$ be given as in (3.1). In addition, we assume that $U \in C^{1}\left(\mathbb{R}^{d}\right)$ and it satisfies

$$
(x, \nabla U(x)) \geq 0,
$$

for all $x \in \mathbb{R}^{d}$. Then, one can construct two $\mathbb{R}^{d}$-valued random variables $X$ and $Y$ such that $X \sim \mu, Y \sim \nu$ and a stochastic domination

$$
|Y| \leq|X| \text { a.s. }
$$

holds. In particular, for every $\psi: \mathbb{R}^{d} \rightarrow \mathbb{R}$, radially symmetric and non-decreasing in $|x|$, we have

$$
E^{\nu}[\psi(Y)] \leq E^{\mu}[\psi(X)] .
$$

Proof. It suffices to prove the theorem under the additional assumption that $\mu$ and $\nu$ have the forms in (2.1), respectively, with $U \in C_{b}^{\infty}\left(\mathbb{R}^{d}\right)$ and radially symmetric $V \in C_{b}^{\infty}\left(\mathbb{R}^{d}\right)$. Define $\tilde{V}:[0, \infty) \rightarrow \mathbb{R}$ such that $\tilde{V}(|x|)=V(x)$. By approximation we can further assume that $\left|\tilde{V}^{\prime}(r) / r\right|$ is bounded on $(0, \infty)$. 
Choosing an $O(d)$-valued function $\left(p_{i j}(x)\right)_{1 \leq i, j \leq d}$ such that $p_{i 1}(x)=x^{i} /|x|(x \neq$ $0)$ and $\delta_{i 1}(x=0)$, we consider a pair of stochastic differential equations:

$$
\begin{aligned}
d X_{t} & =-\frac{1}{2} \nabla V\left(X_{t}\right) d t+p\left(X_{t}\right) d w_{t}, \\
d Y_{t} & =-\frac{1}{2}\left\{\nabla V\left(Y_{t}\right)+\nabla U\left(Y_{t}\right)\right\} d t+p\left(Y_{t}\right) d w_{t},
\end{aligned}
$$

with a common $d$-dimensional Brownian motion $w_{t}=\left(w_{t}^{i}\right)_{i=1}^{d}$. Then both processes $X_{t}$ and $Y_{t}$ are ergodic and their unique invariant measures are $\mu$ and $\nu$, respectively. By Itô's formula applied for $x_{t}=\left|X_{t}\right|^{2}$ and $y_{t}=\left|Y_{t}\right|^{2}$, we have

$$
\begin{aligned}
d x_{t} & =\left\{-\tilde{V}^{\prime}\left(\sqrt{x_{t}}\right) \sqrt{x_{t}}+d\right\} d t+2 \sqrt{x_{t}} d w_{t}^{1}, \\
d y_{t} & =\left\{-\tilde{V}^{\prime}\left(\sqrt{y_{t}}\right) \sqrt{y_{t}}-\left(Y_{t}, \nabla U\left(Y_{t}\right)\right)+d\right\} d t+2 \sqrt{y_{t}} d w_{t}^{1} .
\end{aligned}
$$

Now, condition (3.2) ensures, with the help of the comparison theorem for one dimensional stochastic differential equations (see Chapter VI of [1]) that, if $y_{0} \leq x_{0}$, then $y_{t} \leq x_{t}$ and therefore $\left|Y_{t}\right| \leq\left|X_{t}\right|$ for all $t \geq 0$ a.s.; note that the pathwise uniqueness holds for the stochastic differential equation for $x_{t}$ by YamadaWatanabe's theorem. The ergodicity completes the proof.

Remark 3.4. (1) If the symmetrization $\tilde{U}$ of $U$ defined by

$$
e^{-\tilde{U}(x)}=\int_{S^{d-1}} e^{-U(\sigma x)} d \sigma, \quad x \in \mathbb{R}^{d},
$$

satisfies condition (3.2), then (3.3) holds, where $d \sigma$ stands for the uniform measure on $S^{d-1}=\{\sigma ;|\sigma|=1\}$. Indeed, we have $E^{\nu}[\psi(Y)]=E^{\tilde{\nu}}[\psi(Y)]$ for $\tilde{\nu}$ determined from $\tilde{U}$.

(2) If $\psi: \mathbb{R}^{d} \rightarrow \mathbb{R}$ is radially symmetric and convex, it is non-decreasing in $|x|$.

(3) At least when $d=1,(3.3)$ can be seen directly from the FKG inequality, 8. Note that, when $d=1$, condition (3.2) is equivalent to the fact that $U$ is non-decreasing for $x \geq 0$ and non-increasing for $x \leq 0$.

Corollary 3.5. Let $U \in C^{2}\left(\mathbb{R}^{d}\right)$ be convex and attaining its minimal value at 0 , and let $\psi: \mathbb{R}^{d} \rightarrow \mathbb{R}$ be radially symmetric and non-decreasing in $|x|$. Then, we have

$$
E^{\nu}[\psi(Y)] \leq E^{\mu}[\psi(X)] .
$$

Proof. Since $\nabla U(0)=0$, we have $(x, \nabla U(x))=(x$, Hess $U(\hat{x}) x) \geq 0$ for some $\hat{x}$ by the mean value theorem. Hence, condition (3.2) holds.

If $\mu$ is centered Gaussian and $v \in \mathbb{R}^{d}$ is an eigenvector of its covariance matrix, we have the following theorem:

Theorem 3.6. Let $\mu \in \mathcal{P}\left(\mathbb{R}^{d}\right)$ be a centered Gaussian measure with covariance matrix $A^{-1}$ and let $\nu \in \mathcal{P}\left(\mathbb{R}^{d}\right)$ be determined as in (3.1) from $U$ and $\mu$. Let $e \in \mathbb{R}^{d}$ be an eigenvector of $A$. Then, if $U \in C^{1}\left(\mathbb{R}^{d}\right)$ satisfies

$$
(e, \nabla U(x))(e, x) \geq 0,
$$

for all $x \in \mathbb{R}^{d}$, one can construct two $\mathbb{R}^{d}$-valued random variables $X$ and $Y$ such that $X \sim \mu, Y \sim \nu$ and a stochastic domination

$$
|(e, Y)| \leq|(e, X)| \quad \text { a.s. }
$$

holds. In particular, for every $\psi:[0, \infty) \rightarrow \mathbb{R}$, non-decreasing, we have

$$
E^{\nu}[\psi(|(e, Y)|)] \leq E^{\mu}[\psi(|(e, X)|)] .
$$


Proof. We can assume that $U \in C_{b}^{\infty}\left(\mathbb{R}^{d}\right)$ as in the proof of Theorem 3.3 For the eigenvector $e=\left(e_{i}\right)_{i=1}^{d} \in \mathbb{R}^{d}$ of $A$ such that $|e|=1$, choose $p=\left(p_{i j}\right)_{1 \leq i, j \leq d} \in O(d)$ in such a way that $p_{i 1}=e_{i}, 1 \leq i \leq d$, and consider a pair of stochastic differential equations on $\mathbb{R}^{d}$ :

$$
\begin{aligned}
d X_{t} & =-\frac{1}{2} A X_{t} d t+p d w_{t}, \\
d Y_{t} & =-\frac{1}{2}\left\{A Y_{t}+\nabla U\left(Y_{t}\right)\right\} d t+p d w_{t},
\end{aligned}
$$

with a common $d$-dimensional Brownian motion $w_{t}=\left(w_{t}^{i}\right)_{i=1}^{d}$. Then both processes $X_{t}$ and $Y_{t}$ are ergodic, and their unique invariant measures are $\mu$ and $\nu$, respectively. Noting that $\left(e, p w_{t}\right)=w_{t}^{1}$, by Itô's formula applied for $x_{t}=\left(e, X_{t}\right)^{2}$ and $y_{t}=$ $\left(e, Y_{t}\right)^{2}$, we have

$$
\begin{aligned}
d x_{t} & =\left\{-\lambda x_{t}+d\right\} d t+2 \sqrt{x_{t}} d w_{t}^{1}, \\
d y_{t} & =\left\{-\lambda y_{t}-\left(e, \nabla U\left(Y_{t}\right)\right)\left(e, Y_{t}\right)+d\right\} d t+2 \sqrt{y_{t}} d w_{t}^{1},
\end{aligned}
$$

where $\lambda>0$ is the eigenvalue of $A$ corresponding to $e$ : $A e=\lambda e$. Now, condition (3.4) ensures, with the help of the comparison theorem for one dimensional stochastic differential equations that, if $y_{0} \leq x_{0}$, then $y_{t} \leq x_{t}$ and therefore $\left|\left(e, Y_{t}\right)\right| \leq\left|\left(e, X_{t}\right)\right|$ for all $t \geq 0$ a.s. The ergodicity completes the proof.

Remark 3.7. (1) Condition (3.4) holds for any $e \in \mathbb{R}^{d}$, if $U$ is radially symmetric and non-decreasing in $|x|$ or if $\nabla U$ has a form $\nabla U(x)=f(x) x /|x|$ with $f: \mathbb{R}^{d} \rightarrow[0, \infty)$.

(2) If $U$ is radially symmetric and non-decreasing in $|x|$, then one can realize a stochastic domination: $[(e, Y)]_{+} \leq[(e, X)]_{+}$a.s. with $X \sim \mu, Y \sim \nu$. Compare this with Theorem 3.2 .

\section{An extension to Double-Well potentials}

We propose some extension of Brascamp-Lieb like inequalities to a genuinely non-convex potential, including a potential of double-well type when $d=1$.

Let $\mu \in \mathcal{P}\left(\mathbb{R}^{d}\right)$ be radially symmetric satisfying $0<\mu(D)<1$, where $D=\{x \in$ $\left.\mathbb{R}^{d} ;|x|<1\right\}$. We consider a potential $U_{\epsilon}, \epsilon \in \mathbb{R}$, of the form $U_{\epsilon}(x)=U_{0}(x)+\epsilon W(x)$, where $U_{0}$ satisfies the condition (3.2): $\left(x, \nabla U_{0}(x)\right) \geq 0$ together with $U_{0} \equiv 0$ on $\bar{D}, U_{0} \geq 0$ on $\mathbb{R}^{d}, \mu\left(\left\{x ; U_{0}(x)>0\right\}\right)>0$, and $W$ is a bounded and measurable function on $\mathbb{R}^{d}$ such that $W \equiv 0$ on $D^{c} \equiv \mathbb{R}^{d} \backslash D$. Define $\nu_{\epsilon} \in \mathcal{P}\left(\mathbb{R}^{d}\right)$ by

$$
\nu_{\epsilon}(d x)=\frac{1}{Z_{\epsilon}} e^{-U_{\epsilon}(x)} \mu(d x) .
$$

Corollary 4.1. For $|\epsilon|$ sufficiently small, there exists $c=c_{\epsilon}<1$ such that

$$
E^{\nu_{\epsilon}}[\psi(|Y|)] \leq c E^{\mu}[\psi(|X|)]
$$

for every $\psi:[0, \infty) \rightarrow \mathbb{R}$, which is 0 on $[0,1]$ and non-decreasing for $x \geq 1$.

Proof. Let $\tilde{\mu}=\mu\left(\cdot \mid D^{c}\right)$ and

$$
\tilde{\nu}(d x)=\frac{1}{Z_{\tilde{\nu}}} e^{-U_{0}(x)} \tilde{\mu}(d x)\left(=\nu_{\epsilon}\left(\cdot \mid D^{c}\right)\right) .
$$

Then, for $\psi \equiv 0$ on $[0,1]$, we have

$$
\begin{aligned}
& E^{\mu}[\psi(|X|)]=\mu\left(D^{c}\right) E^{\tilde{\mu}}[\psi(|\tilde{X}|)], \\
& E^{\nu_{\epsilon}}[\psi(|Y|)]=\nu_{\epsilon}\left(D^{c}\right) E^{\tilde{\nu}}[\psi(|\tilde{Y}|)],
\end{aligned}
$$


where $X \sim \mu, Y \sim \nu_{\epsilon}, \tilde{X} \sim \tilde{\mu}$ and $\tilde{Y} \sim \tilde{\nu}$. However, since $E^{\tilde{\nu}}[\psi(|\tilde{Y}|)] \leq E^{\tilde{\mu}}[\psi(|\tilde{X}|)]$ by (3.3), (4.1) holds with

$$
c_{\epsilon}=\frac{\nu_{\epsilon}\left(D^{c}\right)}{\mu\left(D^{c}\right)}=\frac{M}{\mu\left(D^{c}\right)\left\{M+\int_{D} e^{-\epsilon W(x)} \mu(d x)\right\}},
$$

where $M=\int_{D^{c}} e^{-U_{0}(x)} \mu(d x)$. The conditions on $\mu$ and $U_{0}$ imply $M<\mu\left(D^{c}\right)$ so that we have $c_{0}<1$. Moreover the dominated convergence theorem shows that $c_{\epsilon}$ is continuous in $\epsilon \in \mathbb{R}$. This proves the conclusion.

Remark 4.2. The conclusion of Corollary 4.1 does not imply the stochastic domination, which seems invalid for a potential of double-well type in general.

\section{REFERENCES}

1. L. Ambrosio, G. Buttazzo, L.A. Caffarelli, C. Villani and Y. Brenier, Optimal transportation and applications, Martina Franca, Italy 2001, edited by L.A. Caffarelli and S. Salsa, Lecture Notes in Math., 1813 (2003). MR2006302 (2004m:49007)

2. D. Bakry and D. Michel, Sur les inégalités $F K G$, Séminaire de Probabilités, XXVI, pp. 170-188, Lecture Notes in Math., 1526 (1992). MR1231994 (94k:60025)

3. H.J. Brascamp And E.H. Lieb, On extensions of the Brunn-Minkowski and Prékopa-Leindler theorems, including inequalities for log concave functions, and with an application to the diffusion equation, J. Funct. Anal., 22 (1976), pp. 366-389. MR0450480 (56:8774)

4. L.A. CAFFarelLI, Monotonicity properties of optimal transportation and the FKG and related inequalities, Commun. Math. Phys., 214 (2000), pp. 547-563; Erratum, Commun. Math. Phys., 225 (2002), pp. 449-450. MR.1800860 (2002c:60029) MR1889232(2003b:60031)

5. G. Da Prato and J. ZabczyK, Ergodicity for infinite dimensional systems, London Math. Soc. Lect. Note Series, 229, Cambridge Univ. Press, 1996. MR.1417491 (97k:60165)

6. C.M. Fortuin, P.W. Kasteleyn, J. Ginibre, Correlation inequalities on some partially ordered sets, Commun. Math. Phys., 22 (1971), pp. 90-103. MR0309498 (46:8607)

7. G. Giacomin, On stochastic domination in the Brascamp-Lieb framework, Math. Proc. Cambridge Philos. Soc., 134 (2003), pp. 507-514. MR1981215 (2004d:60045)

8. Y. HARIYA, private communication, 2005, Oct.

9. R. Holley, Remarks on the FKG inequalities, Commun. Math. Phys., 36 (1974), pp. 227-231. MR0341552(49:6300)

10. K. ICHIHARA AND H. Kunita, A classification of the second order degenerate elliptic operators and its probabilistic characterization, Z. Wahr. verw. Geb., 30 (1974), pp. 235-254; Supplements and corrections, Z. Wahr. verw. Geb., 39 (1977), pp. 81-84. MR0381007 (52:1904) MR.0488328 (58:7877)

11. N. IKedA AND S. Watanabe, Stochastic differential equations and diffusion processes, 2nd edition, North-Holland, Amsterdam (Kodansha Ltd., Tokyo), 1989. MR1011252 (90m:60069)

12. T.M. LiggetT, Interacting Particle Systems, Springer, 1985. MR0776231 (86e:60089)

13. C.J. Preston, A generalization of the FKG inequalities, Commun. Math. Phys., 36 (1974), pp. 233-241. MR0341553 (49:6301)

14. D.W. Stroock, Probability theory, an analytic view, Cambridge Univ. Press, 1993. MR:1267569 (95f:60003)

Graduate School of Mathematical Sciences, The University of Tokyo, Komaba, TOKYO 153-8914, JAPAN

E-mail address: funaki@ms.u-tokyo.ac.jp

Graduate School of Mathematical Sciences, The University of Tokyo, Komaba, TOKYO 153-8914, JAPAN

Current address: Lehman Brothers Japan Inc., Roppongi Hills, Tokyo

E-mail address: kou.toukairin@lehman.com 